\title{
PD+ Based Trajectory Tracking of the Underactuated Quadrotor Platform using Dual Quaternions*
}

\author{
Tor-Aleksander Johansen, Tom Stian Andersen and Raymond Kristiansen
}

\begin{abstract}
We address the problem of state feedback trajectory tracking of the underactuated quadrotor platform in the dual quaternion framework through a PD+ tracking controller. The control law negates the need of generating a desired attitude trajectory as the translational error is mapped directly onto the rotational actuators through a virtual frame. More precisely, we show uniform practical asymptotic stability of the equilibrium points for the closed-loop system without the presence of disturbances. Simulation results demonstrate the performance of the control law and highlight future work.
\end{abstract}

\section{INTRODUCTION}

Pose control of a rigid body in three dimensional space is an important challenge with broad impact to a number of mechanical systems including, but not limited to; satellites, autonomous underwater vehicles, unmanned aerial vehicles and robot manipulators [1]. The commonly used Newton-Euler equations completely describe the motion of a rigid body in six-degrees-of-freedom (6-DOF), however the rotational and translational movement is often considered separately, thus control algorithms are designed separately. Concurrent position and attitude control is especially relevant in applications such as formation flying, aerial towing, nearearth environment inspection and spacecraft rendezvous and docking. 6-DOF control design often utilize homogeneous transformation matrices, however, dual quaternions is the most compact and efficient way to express motion in three dimensional space [2], [3] and the re-normalization of the unit quaternion is easier than that of the rotation matrix. The main disadvantage is that the unit dual quaternion group is endowed with a double representation of every pose in the configuration space, and control laws designed neglecting this fact will excibit the unwinding phenomenon [4]. This may be countered through a hybrid control scheme [4] or alternativly using the intermediate quaternion approach desccribed in [5]. Quadrotor trajectory tracking has during the last few years received much attention and control design approaches can, for the most part, be separated into two main categories [6]; hierarchical controllers and dynamic extension controllers. Hierarchical controllers rely on an assumption on time-scale separation between the rotational and translational dynamics; one outer controller solves the "slow" translational dynamics by defining the desired attitude trajectory while

\footnotetext{
*This research has been funded by the Norwegian Research Council and is part of the Sikrere logistikk fra fartøy med ubemannet logistikkhelikopter project 282317

All authors are with the Department of Electrical Engineering, UiT The Arctic University of Norway, 8505 Narvik, Norway \{tor-aleksander.johansen, tom.s.andersen, raymond.kristiansen\}aut.no
}

an inner controller solves the "faster" rotational dynamics aligning the body with the desired thrust direction -cf. [7]. Dynamic extension controllers rely on an assumption of a simplified dynamic model allowing for exact linearization of the translational dynamics through differentiation [8]. As it is noted in [6]; the former approach suffers a singularity when the desired thrust direction vanishes, resulting in loss of controllability. The latter approach involves implementation challenges due to the fact that the control input relies on the angular acceleration and jerk of the motors, thus making it necessary to take into account the motor's dynamics in control design. The recent years have seen the emergence of numerous applications of the dual quaternion framework to trajectory tracking purposes, especially in the field of spacecraft trajectory tracking - $c f$. [9] and references therein. Despite this, exploitations of the framework for underactuated systems are few; in [10] dual quaternions is used for modeling and control of an unmanned aerial manipulator consisting of a quadrotor serially coupled with a three-link manipulator, however, dual quaternions are only used for kinematic control and quadrotor dynamics in terms of dual quaternions is not considered. In [11] and [12] the authors present a hierarchical control law for quadrotor stabilization and aerial manipulator tracking. The dual quaternion logarithm is used to generate the desired force vector and subsequently the desired attitude trajectory, however, they do not consider Coriolis acceleration or centrifugal acceleration in their dynamics model.

Applying the classic PD+ controller [13] we develop a state feedback nonlinear control law based on the dual quaternion framework that neither takes the hierarchical form nor relies on dynamic extension to solve the tracking problem for an underactuated system. Utilizing the compact structurepreserving way dual quaternions express rigid body configuration we impose a virtual structure on the system. This allows it to track a desired position trajectory without the explicit construction of a desired attitude trajectory as with the hierarchical form. The approach is inspired by [14] and reminiscent of the work in [15] where a similar approach is used to cancel out unwanted zero dynamics. We show that the origin of the system in closed-loop with the proposed control law is uniformly practically asymptotically stable.

\section{PRELIMINARIES}

\section{A. Notation and reference frames}

Scalar values are denoted in normal face, vectors in lowercase boldface while matrices are written in capital boldface letters. The time derivative is denoted as $\dot{\mathbf{x}}=\frac{d \mathbf{x}}{d t}$, the 
Euclidean norm is denoted as $\|\cdot\|$ while the supremum norm is denoted as $|\cdot|_{\infty}$. Note that $\mathbf{I}_{n \times n}$ denotes an $n \times n$ identity matrix while $\mathbf{0}_{n \times m}$ denotes an $n \times m$ matrix of zeros. Vectors are decomposed in different reference frames denoted by superscripts; $\mathcal{F}^{b}$ is the body frame defined with its x-axis pointing outwards between two of the quadrotors motors, the z-axis is pointing downward and its y-axis completing the right-hand system. $\mathcal{F}^{d}$ denotes the desired frame and $\mathcal{F}^{n}$ is the standard North-East-Down (NED) frame which is assumed to be inertial. The rotation matrix from $\mathcal{F}^{b}$ to $\mathcal{F}^{n}$ is denoted as $\mathbf{R}_{b}^{n} \in S O(3)$, where

$$
S O(3):=\left\{\mathbf{R} \in \mathbb{R}^{3 \times 3}: \mathbf{R}^{\top} \mathbf{R}=\mathbf{I}_{3 \times 3}, \operatorname{det}(\mathbf{R})=1\right\}
$$

is the special orthogonal group. In this work we use unit quaternions to parametrize $S O(3)$, and the equivalent attitude quaternion representing rotations from $\mathcal{F}^{b}$ to $\mathcal{F}^{n}$ is denoted as $\mathbf{q}_{n, b}$. The set of quaternions is defined as $\mathbb{H}:=\{\mathbf{q}=$ $\left.\left[\eta \varepsilon^{\top}\right]^{\top}: \eta \in \mathbb{R}, \varepsilon \in \mathbb{R}^{3}\right\}$ with $\eta$ and $\varepsilon$ denoted as the scalar and the vector part of the quaternion respectivly. The set of unit quaternions is defined as $\mathbb{H}_{u}:=\{\mathbf{q} \in \mathbb{H}:\|\mathbf{q}\|=1\}$. Vectors in $\mathbb{R}^{3}$ can be represented using pure quaternions, $\mathbf{q} \in \mathbb{H}_{p}=\{\mathbf{q} \in \mathbb{H}: \eta=0\}$, by a trivial isomorphism. Unit quaternions topologically form the $3-$ sphere $S^{3}$ in $\mathbb{R}^{4}$,

$$
S^{3}:=\left\{\mathbf{q} \in \mathbb{R}^{4}:\|\mathbf{q}\|=1\right\} .
$$

The homogeneous transformation matrix from $\mathcal{F}^{b}$ to $\mathcal{F}^{n}$ is denoted as $\mathbf{H}_{b}^{n} \in S E(3)$, where

$$
S E(3):=\left\{\mathbf{H} \in \mathbb{R}^{4 \times 4}: \mathbf{H}=\left[\begin{array}{cc}
\mathbf{R} & \mathbf{p} \\
\mathbf{0}_{1 \times 3} & 1
\end{array}\right], \mathbf{R} \in S O(3), \mathbf{p} \in \mathbb{R}^{3}\right\}_{(3)}
$$

is the group of proper Euclidean motion in three dimensional space. In this work we will use unit dual quaternions to parametrize $S E(3)$, and the equivalent pose dual quaternion is denoted $\hat{\mathbf{q}}_{n, b}$. The set of dual quaternions is defined as $\mathbb{D H}:=\left\{\hat{\mathbf{q}}=\mathbf{q}_{p}+\epsilon \mathbf{q}_{d}: \mathbf{q}_{p}, \mathbf{q}_{d} \in \mathbb{H}\right\}$ where $\mathbf{q}_{p}$ and $\mathbf{q}_{d}$ is denoted as the primary and dual part respectively while $\epsilon$ is the dual operator associated with dual numbers, i.e. $\epsilon:=$ $\left\{\epsilon \neq 0, \epsilon^{2}=0\right\}$. The set of unit dual quaternions is defined as $\mathbb{D H}_{u}:=\left\{\hat{\mathbf{q}} \in \mathbb{D H}: \mathbf{q}_{p} \in \mathbb{H}_{u}, \mathbf{q}_{p} \otimes \mathbf{q}_{d}^{*}+\mathbf{q}_{d} \otimes \mathbf{q}_{p}^{*}=0\right\}$ and vectors in $\mathbb{R}^{6}$ can be represented using pure dual quaternions, $\hat{\mathbf{q}} \in \mathbb{D H}_{p}=\left\{\hat{\mathbf{q}} \in \mathbb{D H}: \mathbf{q}_{p}, \mathbf{q}_{d} \in \mathbb{H}_{p}\right\}$. Unit dual quaternions form the group $S^{3} \ltimes \mathbb{R}^{3}$ under multiplication which double covers $S E(3)$, such that

$$
S^{3} \ltimes \mathbb{R}^{3}:=\left\{\hat{\mathbf{q}} \in \mathbb{R}^{8}: \mathbf{q}_{p} \in S^{3}, \mathbf{q}_{d} \in \mathbb{R}^{4}\right\} .
$$

Angular velocity is generally denoted $\boldsymbol{\omega}_{b, c}^{a} \in \mathbb{R}^{3}$, ie. the angular velocity of $\mathcal{F}^{c}$ relative $\mathcal{F}^{b}$ referenced in $\mathcal{F}^{a}$. For any arbitrary vectors $\mathbf{v}_{1}, \mathbf{v}_{2} \in \mathbb{R}^{3}$, we denote the cross-product operator as $\mathbf{S}\left(\mathbf{v}_{1}\right) \mathbf{v}_{2}=\mathbf{v}_{1} \times \mathbf{v}_{2}$. A function $\alpha: \mathbb{R}_{0 \leq} \rightarrow$ $\mathbb{R}_{0 \leq}$ is of class $\mathcal{K}$ if $\alpha$ is strictly increasing, continuous and $\alpha(0)=0$. Moreover, $\alpha$ is of class $\mathcal{K}_{\infty}$ if, in addition, it is unbounded.

\section{B. Quaternions and dual quaternions}

We briefly state some useful concepts for quaternions and dual quaternions, a more comprehensive presentation can be found in literatur -c.f. [9], [16], [17]. The product of two quaternions, $\mathbf{p}$ and $\mathbf{q}$, is defined as

$$
\mathbf{p} \otimes \mathbf{q}:=\left[\begin{array}{c}
\eta_{p} \eta_{q}-\varepsilon_{p}^{\top} \varepsilon_{q} \\
\eta_{p} \varepsilon_{q}+\eta_{q} \varepsilon_{p}+\mathbf{S}\left(\varepsilon_{p}\right) \varepsilon_{q}
\end{array}\right],
$$

while the quaternion conjugate is given as $\mathbf{q}^{*}:=\left[\eta-\varepsilon^{\top}\right]^{\top}$. The rigid body attitude kinematics is modeled by the differential equation

$$
\dot{\mathbf{q}}_{n, b}=\mathbf{T}\left(\mathbf{q}_{n, b}\right) \boldsymbol{\omega}_{n, b}^{b}
$$

where $\dot{\mathbf{q}}_{n, b} \in \mathbb{R}^{4}, \boldsymbol{\omega}_{n, b}^{b} \in \mathbb{R}^{3}$ and $\mathbf{T}\left(\mathbf{q}_{n, b}\right) \in \mathbb{R}^{4 \times 4}$ is defined as

$$
\mathbf{T}\left(\mathbf{q}_{n, b}\right):=\frac{1}{2}\left[\begin{array}{c}
-\varepsilon^{\top} \\
\eta \mathbf{I}_{3 \times 3}+\mathbf{S}(\varepsilon)
\end{array}\right] .
$$

For two pure quaternions, $\mathbf{v}, \mathbf{u} \in \mathbb{R}^{4}$, the cross product is equal to the cross product between the vectors in $\mathbb{R}^{3}$, such that

$$
\mathbf{q} \times \mathbf{p}=\left[\begin{array}{cc}
0 & \mathbf{0}_{1 \times 3} \\
\mathbf{0}_{3 \times 1} & \mathbf{S}\left(\varepsilon_{p}\right)
\end{array}\right] \mathbf{q}:=\mathbf{S}_{q}(\mathbf{p}) \mathbf{q} .
$$

The product of two dual quaternions is calculated as

$$
\hat{\mathbf{q}} \otimes \hat{\mathbf{p}}=\mathbf{q}_{p} \otimes \mathbf{p}_{p}+\epsilon\left(\mathbf{q}_{p} \otimes \mathbf{p}_{d}+\mathbf{q}_{d} \otimes \mathbf{p}_{p}\right)
$$

while the dual quaternion conjugate is defined as ${ }^{1} \hat{\mathbf{q}}^{*}=\mathbf{q}_{p}^{*}+$ $\epsilon \mathbf{q}_{d}^{*}$. For two pure dual quaternions, $\hat{\mathbf{v}}, \hat{\mathbf{u}} \in \mathbb{R}^{8}$, the cross product is defined as

$$
\begin{aligned}
\hat{\mathbf{v}} \times \hat{\mathbf{u}} & =\mathbf{v}_{p} \times \mathbf{u}_{p}+\epsilon\left(\mathbf{v}_{p} \times \mathbf{u}_{d}+\mathbf{v}_{d} \times \mathbf{u}_{p}\right) \\
& =\left[\begin{array}{cc}
\mathbf{S}_{q}\left(\mathbf{v}_{p}\right) & 0 \\
\mathbf{S}_{q}\left(\mathbf{v}_{d}\right) & \mathbf{S}_{q}\left(\mathbf{v}_{p}\right)
\end{array}\right] \hat{\mathbf{u}}:=\hat{\mathbf{S}}(\hat{\mathbf{v}}) \hat{\mathbf{u}} .
\end{aligned}
$$

\section{MAIN RESULT}

In this section we present the main result of this work. For the sake of brevity explicit statements on the use of isomorphisms between $\mathbb{R}^{6}$ and $\mathbb{R}^{8}$ has been omitted.

\section{A. Modelling}

The quadrotor is assumed to be a rigid body, thus the pose of the quadrotor may be represented by an attitude quaternion and a translation vector using the compact dual quaternion framework as

$$
\hat{\mathbf{q}}_{n, b}=\mathbf{q}_{n, b}+\epsilon \frac{1}{2} \mathbf{p}^{n} \otimes \mathbf{q}_{n, b}=\mathbf{q}_{n, b}+\epsilon \frac{1}{2} \mathbf{q}_{n, b} \otimes \mathbf{p}^{b}
$$

where $\mathbf{p}^{n}, \mathbf{p}^{b} \in \mathbb{H}_{p}$ is the quadrotor position expressed in $\mathcal{F}^{n}$ and $\mathcal{F}^{b}$ respectively. The quadrotor kinematics can then be modeled in the dual quaternion framework by the differential equation

$$
\dot{\hat{\mathbf{q}}}_{n, b}=\hat{\mathbf{T}}\left(\hat{\mathbf{q}}_{n, b}\right) \hat{\boldsymbol{\omega}}_{n, b}^{b}
$$

where $\dot{\hat{\mathbf{q}}}_{n, b} \in \mathbb{R}^{8}, \hat{\boldsymbol{\omega}}_{n, b}^{b} \in \mathbb{R}^{6}$ is the dual velocity of the quadrotor, and $\hat{\mathbf{T}}\left(\hat{\mathbf{q}}_{n, b}\right) \in \mathbb{R}^{8 \times 6}$ is defined as

$$
\hat{\mathbf{T}}\left(\hat{\mathbf{q}}_{n, b}\right):=\left[\begin{array}{cc}
\mathbf{T}\left(\mathbf{q}_{p}\right) & \mathbf{0}_{4 \times 3} \\
\mathbf{T}\left(\mathbf{q}_{d}\right) & \mathbf{T}\left(\mathbf{q}_{p}\right)
\end{array}\right]
$$

\footnotetext{
${ }^{1}$ There excists several conjugates for the dual quaternion [9], only one of which we use in this work.
} 
with $\mathbf{T}(\cdot)$ defined in (7). The dual velocity of the quadrotor is defined as

$$
\hat{\boldsymbol{\omega}}_{n, b}^{b}=\boldsymbol{\omega}_{n, b}^{b}+\epsilon \mathbf{v}^{b}
$$

where $\mathbf{v}^{b} \in \mathbb{R}^{3}$ is the translational velocity expressed in $\mathcal{F}^{b}$. Expressing the dual momentum, i.e. the co-screw consisting of linear and angular momentum -cf. [9], as a element of $\mathbb{R}^{6}$ the dynamic model of a rigid body can be derived as

$$
\hat{\mathbf{M}}^{b} \dot{\hat{\boldsymbol{\omega}}}_{n, b}^{b}=\hat{\mathbf{f}}^{b}-\hat{\mathbf{S}}\left(\hat{\boldsymbol{\omega}}_{n, b}^{b}\right) \hat{\mathbf{M}}^{b} \hat{\boldsymbol{\omega}}_{n, b}^{b}
$$

where

$$
\hat{\mathbf{M}}^{b}=\left[\begin{array}{cc}
\mathbf{0}_{3 \times 3} & m \mathbf{I}_{3} \\
\mathbf{J}^{b} & \mathbf{0}_{3 \times 3}
\end{array}\right]
$$

is the dual inertia matrix and $\mathbf{J}^{b}=\operatorname{diag}\left\{\mathrm{J}_{\mathrm{xx}}, \mathrm{J}_{\mathrm{yy}}, \mathrm{J}_{\mathrm{zz}}\right\}$ is the inertia matrix assumed to be diagonal. The variable $\hat{\mathbf{f}}^{b}$ is the dual forces acting on the rigid body. In this work we consider that $\hat{\mathbf{f}}^{b}=\hat{\mathbf{f}}_{u}^{b}+\hat{\mathbf{f}}_{g}^{b}$, with $\hat{\mathbf{f}}_{g}^{b}=\mathbf{q}_{n, b}^{*} \otimes \mathbf{f}_{g}^{n} \otimes \mathbf{q}_{n, b}+\epsilon \mathbf{0}$ being the gravitational force and

$$
\hat{\mathbf{f}}_{u}^{b}=\mathbf{f}_{T}^{b}+\epsilon \boldsymbol{\tau}^{b}=\left[\begin{array}{c}
0 \\
0 \\
T
\end{array}\right]+\epsilon\left[\begin{array}{c}
\tau_{x} \\
\tau_{y} \\
\tau_{z}
\end{array}\right]
$$

defined as the actuator forces, where $T$ is the quadrotor thrust and $\tau_{x}, \tau_{y}, \tau_{z}$ are the actuator torques.

\section{B. Problem formulation}

The tracking control problem can be stated as; let $\hat{\mathbf{q}}_{n, d}$ : $\mathbb{R}_{\geq 0} \rightarrow \mathbb{R}^{8}$ be a feasable, two-times continuously differentiable bounded time-varying desired trajectory, such that

$$
\max \left\{\left|\hat{\mathbf{q}}_{\mathrm{n}, \mathrm{d}}\right|_{\infty},\left|\hat{\boldsymbol{\omega}}_{\mathrm{n}, \mathrm{d}}^{\mathrm{d}}\right|_{\infty},\left|\dot{\hat{\boldsymbol{\omega}}}_{\mathrm{n}, \mathrm{d}}^{\mathrm{d}}\right|_{\infty}\right\} \leq \beta_{\mathrm{d}}
$$

Define the tracking error in dual quaternion coordinates as

$$
\begin{array}{r}
\hat{\mathbf{q}}_{e}:=\hat{\mathbf{q}}_{n, d}^{*} \otimes \hat{\mathbf{q}}_{n, b}=\mathbf{q}_{e}+\epsilon \frac{1}{2} \mathbf{q}_{e} \otimes \mathbf{p}_{e}^{b} \\
:=\mathbf{q}_{e, p}+\epsilon \mathbf{q}_{e, d}=\left[\begin{array}{l}
\eta_{e} \\
\boldsymbol{\varepsilon}_{e}
\end{array}\right]+\epsilon\left[\begin{array}{l}
\eta_{e d} \\
\boldsymbol{\varepsilon}_{e d}
\end{array}\right]
\end{array}
$$

and, due to the double cover $S^{3} \ltimes \mathbb{R}^{3}$ of $S E(3)$, define

$$
\hat{\mathbf{q}}_{e \pm}:=\left[\begin{array}{c}
\left(1 \mp \eta_{e}\right) \\
\varepsilon_{e}
\end{array}\right]+\epsilon \frac{1}{2} \mathbf{q}_{e} \otimes \mathbf{p}_{e}^{b}
$$

with error kinematics and dynamics

$$
\begin{aligned}
\dot{\hat{\mathbf{q}}}_{e \pm} & =\hat{\mathbf{T}}_{e}\left(\hat{\mathbf{q}}_{e \pm}\right) \hat{\boldsymbol{\omega}}_{e}^{b} \\
\hat{\mathbf{M}}^{b} \dot{\hat{\boldsymbol{\omega}}}_{e}^{b} & =\hat{\mathbf{f}}_{u}^{b}+\hat{\mathbf{f}}_{g}^{b}-\hat{\mathbf{S}}\left(\hat{\boldsymbol{\omega}}_{n, b}^{b}\right) \hat{\mathbf{M}} \hat{\boldsymbol{\omega}}_{n, b}^{b}-\hat{\mathbf{M}}^{b} \dot{\hat{\boldsymbol{\omega}}}_{n, d}^{b}
\end{aligned}
$$

where $\hat{\boldsymbol{\omega}}_{e}^{b}=\hat{\boldsymbol{\omega}}_{n, b}^{b}-\hat{\boldsymbol{\omega}}_{n, d}^{b}$ and

$$
\hat{\mathbf{T}}_{e}(\hat{\mathbf{q}})=\left[\begin{array}{cc}
\mathbf{T}_{ \pm}\left(\mathbf{q}_{p}\right) & \mathbf{0}_{3 \times 4} \\
\mathbf{T}\left(\mathbf{q}_{d}\right) & \mathbf{T}\left(\mathbf{q}_{p}\right)
\end{array}\right], \quad \mathbf{T}_{ \pm}\left(\mathbf{q}_{p}\right)=\frac{1}{2}\left[\begin{array}{c} 
\pm \varepsilon^{\top} \\
\eta \mathbf{I}_{3 \times 3}+\mathbf{S}(\varepsilon)
\end{array}\right]
$$

Then, design a feedback control law, $\hat{\mathbf{f}}_{u}^{b}$, that stabilizes the origin for the system (21).

Remark 1: $\hat{\boldsymbol{q}}_{n, d}$ is a function of a desired translational trajectory, $\boldsymbol{p}_{d}^{n}$, and a desired attitude trajectory generated by a desired body frame yaw angle, $\psi_{d}$.

Remark 2: Constructing the desired quaternion from a desired yaw angle infact imposes that the system track six degrees of freedom which strictly speaking makes the problem ill defined for an underactuated system. Under this formulation, practical stability of the equilibrium points is the best result achivable.

Remark 3: Following [18], we define two sets $\hat{\mathbf{q}}_{e+} \in$ $S_{e+}^{3} \ltimes \mathbb{R}^{3}:=\left\{\left[1-\eta_{e}, \varepsilon_{e}^{\top}, \mathbf{q}_{e, d}^{\top}\right]^{\top}: \eta_{e} \geq 0, \hat{\mathbf{q}}_{e} \in S^{3} \ltimes \mathbb{R}^{3}\right\}$ and $\hat{\mathbf{q}}_{e-} \in S_{e-}^{3} \ltimes \mathbb{R}^{3}:=\left\{\left[1+\eta_{e}, \varepsilon_{e}^{\top}, \mathbf{q}_{e, d}^{\top}\right]^{\top}: \eta_{e} \leq 0, \hat{\mathbf{q}}_{e} \in\right.$ $\left.S^{3} \ltimes \mathbb{R}^{3}\right\}$. Thus, $\hat{\mathbf{q}}_{e \pm} \in S_{e+}^{3} \ltimes \mathbb{R}^{3} \cup S_{e-}^{3} \ltimes \mathbb{R}^{3}=S_{e}^{3} \ltimes \mathbb{R}^{3}:=$ $\left\{\left[1-\left|\eta_{e}\right|, \boldsymbol{\varepsilon}_{e}^{\top}, \mathbf{q}_{e, d}^{\top}\right]^{\top}: \hat{\mathbf{q}}_{e} \in S^{3} \ltimes \mathbb{R}^{3}\right\}$.

\section{Controller design}

In order to deal with the challenge of underactuation we introduce two virtual reference frames $\mathcal{F}^{v}$ and $\mathcal{F}^{c}$, both represented by dual quaternions as

$$
\hat{\mathbf{q}}_{b, v}=\mathbf{q}_{I}+\epsilon \frac{1}{2} \boldsymbol{\Delta}^{b} \otimes \mathbf{q}_{I}, \hat{\mathbf{q}}_{v, c}=\mathbf{q}_{v, c}+\epsilon \frac{1}{2} \mathbf{q}_{v, c} \otimes \mathbf{0}
$$

where $\mathbf{0} \in \mathbb{H}_{p}$ is the zero vector as a pure quaternion and $\boldsymbol{\Delta}^{b} \in \mathbb{H}_{p}$ is a constant displacement vector defined as $\boldsymbol{\Delta}^{b}=$ $\left[\begin{array}{llll}0 & 0 & 0 & \Delta\end{array}\right]^{\top}$. Using $\mathcal{F}^{v}$ and $\mathcal{F}^{c}$ we compose an augmented system, $\hat{\mathbf{q}}_{n, c}$, defined as

$$
\hat{\mathbf{q}}_{n, c}=\hat{\mathbf{q}}_{n, b} \otimes \hat{\mathbf{q}}_{b, v} \otimes \hat{\mathbf{q}}_{v, c}=\mathbf{q}_{n, c}+\epsilon \frac{1}{2} \mathbf{p}_{c}^{n} \otimes \mathbf{q}_{n, c}
$$

and derive its kinematics

$$
\dot{\hat{\mathbf{q}}}_{n, c}=\frac{1}{2} \hat{\mathbf{q}}_{n, c} \otimes \hat{\boldsymbol{\omega}}_{n, c}^{c}
$$

with $\hat{\boldsymbol{\omega}}_{n, c}^{c}=\hat{\boldsymbol{\omega}}_{n, b}^{c}+\hat{\boldsymbol{\omega}}_{b, v}^{c}+\hat{\boldsymbol{\omega}}_{v, c}^{c}$. Taking the derivative of the composed system dual velocity we find

$$
\dot{\hat{\boldsymbol{\omega}}}_{n, c}^{c}=\hat{\mathbf{q}}_{b, c}^{*} \otimes \dot{\hat{\boldsymbol{\omega}}}_{n, b}^{b} \otimes \hat{\mathbf{q}}_{b, c}+\hat{\mathbf{S}}\left(\hat{\boldsymbol{\omega}}_{n, b}^{c}\right) \hat{\boldsymbol{\omega}}_{b, c}^{c}+\dot{\hat{\boldsymbol{\omega}}}_{v, c}^{c}
$$

which after inserting the dynamics of (15) becomes

$$
\begin{aligned}
\dot{\hat{\boldsymbol{\omega}}}_{n, c}^{c}= & \hat{\mathbf{q}}_{b, c}^{*} \otimes\left(\hat{\mathbf{M}}^{b}\right)^{-1}\left(\hat{\mathbf{f}}_{u}^{b}-\hat{\mathbf{f}}_{g}^{b}-\hat{\boldsymbol{\omega}}_{n, b}^{b} \times \hat{\mathbf{M}}^{b} \hat{\boldsymbol{\omega}}_{n, b}^{b}\right) \otimes \hat{\mathbf{q}}_{b, c} \\
& +\hat{\mathbf{S}}\left(\hat{\boldsymbol{\omega}}_{n, b}^{c}\right) \hat{\boldsymbol{\omega}}_{b, c}^{c}+\dot{\hat{\boldsymbol{\omega}}}_{v, c}^{c} .
\end{aligned}
$$

Isolating and examining the control dual force of the quadrotor, transformed into $\mathcal{F}^{c}$, one finds

$$
\hat{\mathbf{q}}_{b, c}^{*} \otimes\left(\hat{\mathbf{M}}^{b}\right)^{-1} \hat{\mathbf{f}}_{u}^{b} \otimes \hat{\mathbf{q}}_{b, c}=\left[\begin{array}{c}
\mathbf{q}_{b, c}^{*} \otimes\left(\mathbf{J}^{b}\right)^{-1} \boldsymbol{\tau}^{b} \otimes \mathbf{q}_{b, c} \\
{\left[\begin{array}{c}
\Delta \tau_{y} / J_{y y} \\
-\Delta \tau_{x} / J_{x x} \\
T / m
\end{array}\right] \otimes \mathbf{q}_{b, c}}
\end{array}\right] .
$$

The last equation shows that the quadrotor torques have been mapped to produce a force in the new composed system, thus the body will pitch and roll according to the desired acceleration of $\mathcal{F}^{c}$.

Remark 4: The addition of the second virtual frame is used to cancel any pitching/rolling motion made by the quadrotor, which enables the use of the dual quaternion framework for the underactuated tracking problem. 
We now proceed to define a control screw for the composed system

$$
\hat{\mathbf{u}}^{c}=\left[\begin{array}{c}
\dot{\boldsymbol{\omega}}_{v, c}^{c}+\mathbf{q}_{b, c}^{*} \otimes\left[\begin{array}{c}
0 \\
0 \\
\tau_{z} / J_{z z}
\end{array}\right] \otimes \mathbf{q}_{b, c} \\
\mathbf{q}_{b, c}^{*} \otimes\left[\begin{array}{c}
\Delta \tau_{y} / J_{y y} \\
-\Delta \tau_{x} / J_{x x} \\
T / m
\end{array}\right] \otimes \mathbf{q}_{b, c}
\end{array}\right]
$$

and restate equation (27) as

$$
\begin{aligned}
\dot{\hat{\boldsymbol{\omega}}}_{n, c}^{c}= & \hat{\mathbf{u}}^{c}-\hat{\mathbf{q}}_{b, c}^{*} \otimes\left(\hat{\mathbf{M}}^{b}\right)^{-1}\left(\hat{\mathbf{f}}_{g}^{b}+\hat{\boldsymbol{\omega}}_{n, b}^{b} \times \hat{\mathbf{M}}^{b} \hat{\boldsymbol{\omega}}_{n, b}^{b}\right) \otimes \hat{\mathbf{q}}_{b, c} \\
& +\hat{\mathbf{S}}\left(\hat{\boldsymbol{\omega}}_{n, b}^{c}\right) \hat{\boldsymbol{\omega}}_{b, c}^{c}+\hat{\delta}\left(\hat{\mathbf{u}}^{c}\right)
\end{aligned}
$$

where

$$
\hat{\delta}\left(\hat{\mathbf{u}}^{c}\right)=\left[\mathbf{q}_{b, c}^{*} \otimes\left[\begin{array}{c}
\tau_{x} / J_{x x} \\
\tau_{y} / J_{y y} \\
0 \\
\mathbf{0}
\end{array}\right] \otimes \mathbf{q}_{b, c}\right] .
$$

As can be seen in equation (28) the composed augmented system $\hat{\mathbf{q}}_{n, c}$ is fully actuated with regards to the configuration space $S E(3)$, with four real and three virtual actuators. Due to the fact that two rotational actuators have been mapped to the translational error and any rotational motion is cancelled by the virtual frame leaves the system without damping for these two degrees of freedom and damping need to be inserted. In a similar fashion as the way we augmented the original system we augment the desired trajectory $\hat{\mathbf{q}}_{d}$. Defining another virtual frame, $\mathcal{F}^{z}$, similarly as in (23)

$$
\hat{\mathbf{q}}_{d, z}=\mathbf{q}_{I}+\epsilon \frac{1}{2} \boldsymbol{\Delta}^{d} \otimes \mathbf{q}_{I}
$$

where $\boldsymbol{\Delta}^{d}:=\boldsymbol{\Delta}^{b}$. The new desired trajectory can be composed and augmented as

$$
\begin{aligned}
& \hat{\mathbf{q}}_{n, z}=\hat{\mathbf{q}}_{n, d} \otimes \hat{\mathbf{q}}_{d, z}=\mathbf{q}_{n, z}+\epsilon \frac{1}{2} \mathbf{p}_{z}^{n} \otimes \mathbf{q}_{n, z} \\
& \hat{\boldsymbol{\omega}}_{n, z}^{z}=\hat{\mathbf{q}}_{d, z}^{*} \otimes \hat{\boldsymbol{\omega}}_{n, d}^{d} \otimes \hat{\mathbf{q}}_{d, z} \\
& \dot{\hat{\boldsymbol{\omega}}}_{n, z}^{z}=\hat{\mathbf{q}}_{d, z}^{*} \otimes \dot{\hat{\boldsymbol{\omega}}}_{n, d}^{d} \otimes \hat{\mathbf{q}}_{d, z} .
\end{aligned}
$$

Now we state the tracking error as

$$
\hat{\mathbf{q}}_{e}=\hat{\mathbf{q}}_{n, z}^{*} \otimes \hat{\mathbf{q}}_{n, c}:=\mathbf{q}_{e}+\epsilon \frac{1}{2} \mathbf{q}_{e} \otimes \mathbf{p}_{e}^{c}
$$

with error kinematics and dynamics

$$
\begin{aligned}
\dot{\hat{\mathbf{q}}}_{e \pm}= & \hat{\mathbf{T}}_{e}\left(\hat{\mathbf{q}}_{e \pm}\right) \hat{\boldsymbol{\omega}}_{e}^{c} \\
\dot{\hat{\boldsymbol{\omega}}}_{e}^{c}= & \hat{\mathbf{u}}^{c}-\hat{\mathbf{q}}_{b, c}^{*} \otimes\left(\hat{\mathbf{M}}^{b}\right)^{-1}\left(\hat{\mathbf{f}}_{g}^{b}+\hat{\boldsymbol{\omega}}_{n, b}^{b} \times \hat{\mathbf{M}}^{b} \hat{\boldsymbol{\omega}}_{n, b}^{b}\right) \otimes \hat{\mathbf{q}}_{b, c} \\
& +\hat{\mathbf{S}}\left(\hat{\boldsymbol{\omega}}_{n, b}^{c}\right) \hat{\boldsymbol{\omega}}_{b, c}^{c}+\hat{\delta}\left(\hat{\mathbf{u}}^{c}\right)-\dot{\hat{\boldsymbol{\omega}}}_{n, z}^{c}
\end{aligned}
$$

where $\hat{\boldsymbol{\omega}}_{e}^{c}=\hat{\boldsymbol{\omega}}_{n, c}^{c}-\hat{\boldsymbol{\omega}}_{n, z}^{c}$.

The following proposition formalizes the outlined control strategy

Proposition 1: Let the pose of the augmented system be composed as in (24) and the desired trajectory be augmented as in (32). Further, let the error system be defined by (33) and (34) and assume that the body frame has bounded angular velocities, i.e. $\left\|\mathbf{L} \boldsymbol{\omega}_{n, b}^{b}\right\| \leq \gamma_{1}$, with $\mathbf{L}=\operatorname{diag}\left\{\begin{array}{lll}1 & 1 & 0\end{array}\right\}$ and $\gamma_{1} \in \mathbb{R}$. Then, asymptotically stabilizing the origin of the system (34) is equivalent to asymptotically stabilizing the ball of radius $\gamma$ around the origin of system (21).

Proof: Consider the equilibrium point of the system, i.e. $\left(\hat{\mathbf{q}}_{e \pm}, \hat{\boldsymbol{\omega}}_{e}^{c}\right)=(\mathbf{0}, \mathbf{0})$. When the system (34) is stabilized, the primary part of the dual quaternion error (33) is equal to the identity quaternion. This implies

$$
\mathbf{q}_{n, c}=\mathbf{q}_{n, z} \Rightarrow \mathbf{q}_{n, b} \otimes \mathbf{q}_{v, c}=\mathbf{q}_{n, d}
$$

given $\mathbf{q}_{b, v}=\mathbf{q}_{d, z}=\mathbf{q}_{I}$. As mentioned in Remark 1, $\mathbf{q}_{n, d}$ is generated by a desired yaw angle $\psi_{d}$. Using this and the fact that any unit quaternion representing a rigid body's attitude is composed as the product of three quaternions generated by the roll, pitch and yaw angles of that body, we restate (35) as

$$
\mathbf{q}_{n, b}(\psi) \otimes \mathbf{q}_{n, b}(\theta, \phi) \otimes \mathbf{q}_{v, c}=\mathbf{q}_{n, d}\left(\psi_{d}\right)
$$

As mentioned in Remark $4, \mathbf{q}_{v, c}$ is used to cancel out any rolling and pitching motion made by the quadrotor, leading to

$$
\mathbf{q}_{v, c}=\mathbf{q}_{n, b}^{*}(\theta, \phi) \Rightarrow \mathbf{q}_{n, b}(\psi)=\mathbf{q}_{n, d}\left(\psi_{d}\right) .
$$

This implies that the body yaw angle is the desired yaw angle, and further the attitude error between $\mathcal{F}^{d}$ and $\mathcal{F}^{b}$ can be stated as

$$
\mathbf{q}_{n, d}^{*} \otimes \mathbf{q}_{n, b}=\mathbf{q}_{v, c}^{*} .
$$

For the dual part of the dual quaternion error in (33), $\hat{\mathbf{q}}_{e, d}=$ 0,implies

$$
\begin{gathered}
\mathbf{p}_{c}^{n}=\mathbf{p}_{z}^{n} \\
\mathbf{p}^{n}+\mathbf{q}_{n, b} \otimes \boldsymbol{\Delta}^{b} \otimes \mathbf{q}_{n, b}^{*}=\mathbf{p}_{d}^{n}+\mathbf{q}_{n, d} \otimes \boldsymbol{\Delta}^{d} \otimes \mathbf{q}_{n, d}^{*} \\
\mathbf{p}^{n}-\mathbf{p}_{d}^{n}=\mathbf{q}_{n, d} \otimes \boldsymbol{\Delta}^{d} \otimes \mathbf{q}_{n, d}^{*}-\mathbf{q}_{n, b} \otimes \boldsymbol{\Delta}^{b} \otimes \mathbf{q}_{n, b}^{*}
\end{gathered}
$$

which, after inserting relation (37) and the definition of $\boldsymbol{\Delta}^{d}$ becomes

$$
\begin{aligned}
\mathbf{p}_{e}^{n} & =\mathbf{q}_{n, b}(\psi) \otimes \boldsymbol{\Delta}^{b} \otimes \mathbf{q}_{n, b}^{*}(\psi)-\mathbf{q}_{n, b} \otimes \boldsymbol{\Delta}^{b} \otimes \mathbf{q}_{n, b}^{*} \\
& =\boldsymbol{\Delta}^{b}-\mathbf{q}_{n, b} \otimes \boldsymbol{\Delta}^{b} \otimes \mathbf{q}_{n, b}^{*} \\
& =\left\|\boldsymbol{\Delta}^{b}\right\|\left(\mathbf{e}_{3}-\mathbf{q}_{n, b} \otimes \mathbf{e}_{3} \otimes \mathbf{q}_{n, b}^{*}\right)
\end{aligned}
$$

where $\mathbf{e}_{3}=\left[\begin{array}{lll}0 & 0 & 1\end{array}\right]^{\top}$ and we have used the fact that $\boldsymbol{\Delta}^{b}$ is invariant under yaw rotation. Thus, it can be seen that $\left\|\mathbf{p}_{e}^{n}\right\|$ will be less than or equal to $2\left\|\boldsymbol{\Delta}^{b}\right\|$ depending on the orientation of the quadrotor body frame. Further, considering the dual velocity error when the system (34) is stabilized, we have that

$$
\begin{aligned}
\hat{\boldsymbol{\omega}}_{n, c}^{c} & =\hat{\boldsymbol{\omega}}_{n, z}^{c} \\
\hat{\boldsymbol{\omega}}_{n, b}^{c}+\hat{\boldsymbol{\omega}}_{v, c}^{c} & =\hat{\mathbf{q}}_{e}^{*} \otimes \hat{\boldsymbol{\omega}}_{n, z}^{z} \otimes \hat{\mathbf{q}}_{e} \\
\hat{\boldsymbol{\omega}}_{n, b}^{b} & =\hat{\mathbf{q}}_{b, z} \otimes \hat{\boldsymbol{\omega}}_{n, z}^{z} \otimes \hat{\mathbf{q}}_{b, z}^{*}-\hat{\mathbf{q}}_{b, c} \otimes \hat{\boldsymbol{\omega}}_{v, c}^{c} \otimes \hat{\mathbf{q}}_{b, c}^{*} .
\end{aligned}
$$

For the primary part

$$
\begin{aligned}
\boldsymbol{\omega}_{n, b}^{b} & =\mathbf{q}_{d, b}^{*} \otimes \boldsymbol{\omega}_{n, d}^{d} \otimes \mathbf{q}_{d, b}-\mathbf{q}_{b, c} \otimes \boldsymbol{\omega}_{v, c}^{c} \otimes \mathbf{q}_{b, c}^{*} \\
\boldsymbol{\omega}_{d, b}^{b} & =-\mathbf{q}_{b, c} \otimes \boldsymbol{\omega}_{v, c}^{c} \otimes \mathbf{q}_{b, c}^{*}
\end{aligned}
$$

and from (38) we have the relation $\boldsymbol{\omega}_{v, c}^{b}=\boldsymbol{\omega}_{n, d}^{b}-\boldsymbol{\omega}_{n, b}^{b}$ thus $\boldsymbol{\omega}_{d, b}^{b}=\boldsymbol{\omega}_{d, b}^{b}$, which is consistent with reference to Remark 
2 and the mention earlier about the lack of damping. Finally for the dual part we have

$$
\begin{aligned}
\mathbf{v}^{b}= & \mathbf{q}_{b, d} \otimes \mathbf{v}^{d} \otimes \mathbf{q}_{b, d}^{*}+\mathbf{S}_{q}\left(\boldsymbol{\omega}_{n, b}^{b}\right)\left(\boldsymbol{\Delta}^{b}+\mathbf{p}_{e}^{b}\right) \\
& -\mathbf{S}_{q}\left(\boldsymbol{\omega}_{n, b}^{b}\right) \boldsymbol{\Delta}^{b}
\end{aligned}
$$

which after rearranging gives

$$
\mathbf{v}_{e}^{b}=-\mathbf{S}_{q}\left(\boldsymbol{\omega}_{d, b}^{b}\right) \mathbf{p}_{e}^{b} .
$$

Consider now that $\mathbf{q}_{v, c}=\left[\begin{array}{ll}\eta_{c} & \boldsymbol{\varepsilon}_{c}^{\top}\end{array}\right]^{\top}$, then, when the system (34) is stabilized, we have with reference to the original system (21)

$$
\begin{aligned}
\hat{\mathbf{q}}_{e \pm}^{\top} \hat{\mathbf{q}}_{e \pm} & =\left(1 \mp \eta_{c}\right)^{2}+\boldsymbol{\varepsilon}_{c}^{\top} \boldsymbol{\varepsilon}_{c}+\frac{1}{4}\left(\mathbf{p}_{e}^{b}\right)^{\top} \mathbf{p}_{e}^{b} \\
& \leq 2+\|\boldsymbol{\Delta}\|^{2}:=\gamma_{2}
\end{aligned}
$$

and

$$
\begin{aligned}
\left(\hat{\boldsymbol{\omega}}_{e}^{b}\right)^{\top} \hat{\boldsymbol{\omega}}_{e}^{b} & =\left(\boldsymbol{\omega}_{d, b}^{b}\right)^{\top} \boldsymbol{\omega}_{d, b}^{b}+\left(\mathbf{v}_{e}^{b}\right)^{\top} \mathbf{v}_{e}^{b} \\
& \leq\left(\gamma_{1}^{2}+2 \gamma_{1} \beta_{d}+\beta_{d}^{2}\right)\left(1+2\|\boldsymbol{\Delta}\|^{2}\right):=\gamma_{3}
\end{aligned}
$$

Defining $\gamma=\gamma_{2}+\gamma_{3}$ concludes the proof.

Under Proposition 1 the problem formulation in III-B can be restated to designing a feedback control law, $\hat{\mathbf{u}}^{c}$, that stabilizes the origin of (34).

Remark 5: The assumption on boundedness of the body frame angular velocities in Proposition 1 is due to the fact that there is no damping in the system for the angular velocities. As the derivative of the body frame angular velocities is directly mapped to the translational error this assumption can readily be meet through (46) and starting sufficiently close to the desired trajectory.

Remark 6: Under the condition of boundedness of the original desired trajectory, (18), the augmented desired trajectory (32) will also be bounded, i.e.

$$
\max \left\{\left|\hat{\boldsymbol{q}}_{\mathrm{n}, \mathrm{z}}\right|_{\infty},\left|\hat{\boldsymbol{\omega}}_{\mathrm{n}, \mathrm{z}}^{\mathrm{z}}\right|_{\infty},\left|\dot{\hat{\boldsymbol{\omega}}}_{\mathrm{n}, \mathrm{z}}^{\mathrm{z}}\right|_{\infty}\right\} \leq \beta_{\mathrm{z}} .
$$

We omit the proof for brevity, but the result is readily found by direct computation.

Before presenting our main result we present some technical lemmas that will be used later:

Lemma 3.1: Let $\hat{\mathbf{T}}_{e}\left(\hat{\mathbf{q}}_{e \pm}\right)$ be defined in (22), further let $\mathbf{q}_{e \pm}$ be defined as in (20). Then, it holds that

$$
\hat{\mathbf{q}}_{e \pm}^{\top} \hat{\mathbf{T}}_{e}\left(\hat{\mathbf{q}}_{e \pm}\right)=\frac{1}{2}\left[ \pm \varepsilon_{e}^{\top} \quad \frac{1}{2}\left(\mathbf{p}_{e}^{b}\right)^{\top}\right]:=\frac{1}{2} \tilde{\varepsilon}^{\top}
$$

The proof can be found in Appendix A.

Lemma 3.2: Let $\hat{\mathbf{T}}_{e}\left(\hat{\mathbf{q}}_{e \pm}\right)$ be defined as in (22), $\mathbf{q}_{e \pm}$ be defined as in (20) and let $\hat{\boldsymbol{\omega}}_{e}^{c}$ be the error velocity satisfying (34). Define $\Xi=\left[\mathbf{q}_{e \pm}^{\top} \hat{\mathbf{T}}_{e}\left(\hat{\mathbf{q}}_{e \pm}\right)\right]^{\top}$, then

$$
0 \leq\|\Xi\|^{2} \leq \hat{\mathbf{q}}_{e \pm}^{\top} \hat{\mathbf{q}}_{e \pm}
$$

The proof can be found in Appendix B.

We are now ready to present our main result, which establishes uniform asymptotic stability of the closed-loop augmented system under a modified PD+ controller.
Proposition 2: Let $\hat{\boldsymbol{q}}_{e q} \in S_{e}^{3} \ltimes \mathbb{R}^{3}$ and $\operatorname{sgn}\left(\eta_{\mathrm{e}, \mathrm{p}}\left(\mathrm{t}_{0}\right)\right)=$ $\operatorname{sgn}\left(\eta_{\mathrm{e}, \mathrm{p}}(\mathrm{t})\right)$ for all $t \geq t_{0}{ }^{2}$, let the desired trajectory, $\hat{\boldsymbol{q}}_{n, z}$, satisfy (46). Then the equilibrium points $\left(\hat{\boldsymbol{q}}_{e \pm}, \hat{\boldsymbol{\omega}}_{e}^{c}\right)=(\boldsymbol{0}, \boldsymbol{0})$ of the system (34), in closed-loop with the control law

$$
\begin{aligned}
\hat{\boldsymbol{u}}^{c} & =\hat{\boldsymbol{q}}_{b, c}^{*} \otimes\left(\hat{\boldsymbol{M}}^{b}\right)^{-1}\left(\hat{\boldsymbol{f}}_{g}^{b}+\hat{\boldsymbol{S}}\left(\hat{\boldsymbol{\omega}}_{n, b}^{b}\right) \hat{\boldsymbol{M}}^{b} \hat{\boldsymbol{\omega}}_{n, b}^{b}\right) \otimes \hat{\boldsymbol{q}}_{b, c} \\
& -\hat{\boldsymbol{S}}\left(\hat{\boldsymbol{\omega}}_{n, b}^{c}\right) \hat{\boldsymbol{\omega}}_{b, c}^{c}-\hat{\delta}\left(\hat{\boldsymbol{u}}^{c}\right)+\hat{\boldsymbol{q}}_{e}^{*} \otimes \dot{\hat{\boldsymbol{\omega}}}_{n, z}^{z} \otimes \hat{\boldsymbol{q}}_{e}+\hat{\boldsymbol{S}}\left(\hat{\boldsymbol{\omega}}_{n, z}^{c}\right) \hat{\boldsymbol{\omega}}_{e}^{c} \\
& -\boldsymbol{K}_{p} \tilde{\boldsymbol{\varepsilon}}-\boldsymbol{K}_{d} \hat{\boldsymbol{\omega}}_{e}
\end{aligned}
$$

where $\boldsymbol{K}_{p}, \boldsymbol{K}_{d}$ are positive feedback gain matrices, are uniformly asymptotically stable (UAS).

Proof: In the following we only consider, without loss of generality, the positive equilibrium point, i.e. $\hat{\mathbf{q}}_{e q}=\hat{\mathbf{q}}_{e+}$ and $\hat{\mathbf{T}}_{e}\left(\hat{\mathbf{q}}_{e q}\right)=\hat{\mathbf{T}}_{e}\left(\hat{\mathbf{q}}_{e+}\right)$. The closed-loop kinematics and dynamics, resulting from inserting (49) into (34), is

$$
\begin{aligned}
\dot{\hat{\mathbf{q}}}_{e q} & =\hat{\mathbf{T}}_{e}\left(\hat{\mathbf{q}}_{e q}\right) \hat{\boldsymbol{\omega}}_{e}^{c} \\
\dot{\hat{\boldsymbol{\omega}}}_{e}^{c} & =-\mathbf{K}_{p} \tilde{\varepsilon}-\mathbf{K}_{d} \hat{\boldsymbol{\omega}}_{e}^{c}
\end{aligned}
$$

Consider the radially unbounded Lyapunov function candidate

$$
V\left(\hat{\mathbf{q}}_{e q}, \hat{\boldsymbol{\omega}}_{e}^{c}\right)=\hat{\mathbf{q}}_{e q}^{\top} \mathbf{K}_{p} \hat{\mathbf{q}}_{e q}+\frac{1}{2}\left(\hat{\boldsymbol{\omega}}_{e}^{c}\right)^{\top} \hat{\boldsymbol{\omega}}_{e}^{c} .
$$

We show that there exist functions $\underline{\alpha}, \bar{\alpha} \in \mathcal{K}_{\infty}$ such that $\underline{\alpha}(\mathbf{x}) \leq V(\mathbf{x}) \leq \bar{\alpha}(\mathbf{x})$. Defining $\boldsymbol{\chi}=\left[\hat{\mathbf{q}}_{e q}^{\top} \hat{\mathbf{T}}_{e}\left(\hat{\mathbf{q}}_{e q}\right)\left(\hat{\boldsymbol{\omega}}_{e}^{c}\right)^{\top}\right]^{\top}$ and utilizing Lemma 3.2, we obtain

$$
p_{m}\|\chi\|^{2} \leq V\left(\hat{\mathbf{q}}_{e q}, \hat{\boldsymbol{\omega}}_{e}^{c}\right) \leq p_{M}\|\chi\|^{2}
$$

for some $p_{M}>p_{m}>0$. Thus choosing $\underline{\alpha}\left(\hat{\mathbf{q}}_{e q}, \hat{\boldsymbol{\omega}}_{e}^{c}\right)=$ $p_{m}\|\chi\|^{2}$ and $\bar{\alpha}\left(\hat{\mathbf{q}}_{e q}, \hat{\boldsymbol{\omega}}_{e}^{c}\right)=p_{M}\|\boldsymbol{\chi}\|^{2}$ ensures the existence of such functions. Evaluating the time derivative of $V$ along the closed-loop trajectories generated by (50) yields

$$
\begin{aligned}
\dot{V}\left(\hat{\mathbf{q}}_{e q}, \hat{\boldsymbol{\omega}}_{e}^{c}\right)= & 2 \hat{\mathbf{q}}_{e q}^{\top} \hat{\mathbf{K}}_{p} \hat{\mathbf{T}}_{e}\left(\hat{\mathbf{q}}_{e q}\right) \hat{\boldsymbol{\omega}}_{e}^{c} \\
& +\left(\hat{\boldsymbol{\omega}}_{e}^{c}\right)^{\top}\left(-\hat{\mathbf{K}}_{p} \tilde{\boldsymbol{\varepsilon}}-\hat{\mathbf{K}}_{d} \hat{\boldsymbol{\omega}}_{e}^{c}\right) \\
= & \left(\hat{\mathbf{K}}_{p} \tilde{\varepsilon}\right)^{\top} \hat{\boldsymbol{\omega}}_{e}^{c}-\left(\hat{\boldsymbol{\omega}}_{e}^{c}\right)^{\top} \hat{\mathbf{K}}_{p} \tilde{\varepsilon}-\left(\hat{\boldsymbol{\omega}}_{e}^{c}\right)^{\top} \hat{\mathbf{K}}_{d} \hat{\boldsymbol{\omega}}_{e}^{c} \\
= & -\left(\hat{\boldsymbol{\omega}}_{e}^{c}\right)^{\top} \hat{\mathbf{K}}_{d} \hat{\boldsymbol{\omega}}_{e}^{c} \leq 0 .
\end{aligned}
$$

We conclude, by Theorem 4.8 in [19], that the equilibrium point $\left(\hat{\mathbf{q}}_{e q}, \hat{\boldsymbol{\omega}}_{e}^{c}\right)=(\mathbf{0}, \mathbf{0})$ is uniformly stable and the solutions are uniformly bounded.

To show uniform asymptotic stability we invoke Matrosov's theorem, as stated in [13], by introducing the auxiliary function

$$
W\left(\hat{\mathbf{q}}_{e q}, \hat{\boldsymbol{\omega}}_{e}^{c}\right)=\tilde{\boldsymbol{\varepsilon}}^{\top} \hat{\boldsymbol{\omega}}_{e}^{c}=2 \hat{\mathbf{q}}_{e q}^{\top} \hat{\mathbf{T}}_{e}\left(\hat{\mathbf{q}}_{e q}\right) \hat{\boldsymbol{\omega}}_{e}^{c}
$$

which is continuous in both arguments and depends on time through the bounded reference function $\hat{\mathbf{q}}_{d *}$. Differentiation of the auxiliary function yields

$$
\dot{W}=2 \dot{\hat{\mathbf{q}}}_{e q}^{\top} \hat{\mathbf{T}}_{e}\left(\hat{\mathbf{q}}_{e q}\right) \hat{\boldsymbol{\omega}}_{e}^{c}+2 \hat{\mathbf{q}}_{e q}^{\top} \dot{\hat{\mathbf{T}}}_{e}\left(\hat{\mathbf{q}}_{e q}\right) \hat{\boldsymbol{\omega}}_{e}^{c}+\tilde{\boldsymbol{\varepsilon}}^{\top} \dot{\hat{\boldsymbol{\omega}}}_{e}^{c}
$$

\footnotetext{
${ }^{2}$ This assumption can be relaxed by employing a hybrid control strategy for the controller, this is however not the main focus of this work.
} 
and after inserting (50) one can varify that on the set $E=$ $\{\dot{V}=0\}=\left\{\hat{\boldsymbol{\omega}}_{e}^{c}=\mathbf{0}\right\}$,

$$
\dot{W}=-\tilde{\varepsilon}^{\top} \mathbf{K}_{p} \tilde{\varepsilon}
$$

That is, $\dot{W}$ is non-zero definite on $E$. Thus all conditions of Matrosov's theorem are satisfied, and $\left(\hat{\mathbf{q}}_{e \pm}, \hat{\boldsymbol{\omega}}_{e}^{c}\right) \rightarrow(\mathbf{0}, \mathbf{0})$ asymptotically. The proof for the negative equilibrium point, $\hat{\mathbf{q}}_{e q}=\hat{\mathbf{q}}_{e-}$ and $\hat{\mathbf{T}}_{e}\left(\hat{\mathbf{q}}_{e q}\right)=\hat{\mathbf{T}}_{e}\left(\hat{\mathbf{q}}_{e-}\right)$ is performed in the same way. It follows that the dual equilibrium points $\hat{\mathbf{q}}_{e q} \in S_{e}^{3} \ltimes \mathbb{R}^{3}$ are UAS.

Remark 7: By proposition 2 the control law in (49) uniformly stabilizes the error system (34), which under proposition 1 implies that the original error system (21) is uniformly stabilized to a ball of radius $\gamma$ around the origin.

\section{Simulations}

In this section, simulation results for a underactuated quadrotor tracking a trajectory are presented to demonstrate the performance of the presented control law in Proposition 2. The quadrotor model is based upon the UiTRotor quadrotor that have a mass of $1.3 \mathrm{~kg}$, and moments of given

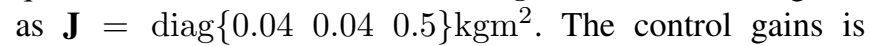
given as $\mathbf{K}_{p}=\operatorname{diag}\left\{\begin{array}{llllllll}1 & 1 & 1 & 1 & 1 & 0.1 & 0.1 & 0.1\end{array}\right\}, \mathbf{K}_{d}=$ $\operatorname{diag}\left\{\begin{array}{lllllllll}1 & 2 & 2 & 2 & 1 & 0.4 & 0.4 & 2\end{array}\right\}$. The initial condition for the quadrotor system is

$$
\begin{aligned}
\hat{\mathbf{q}}_{n, b}\left(t_{0}\right) & =\hat{\mathbf{q}}_{I}+\varepsilon \frac{1}{2} \hat{\mathbf{q}}_{I} \otimes\left[\begin{array}{llll}
0 & 0 & 5 & -5
\end{array}\right]^{\top} \\
\hat{\boldsymbol{\omega}}_{n, b}^{b}\left(t_{0}\right) & =\left[\begin{array}{lllllll}
0 & 0.1 & 0.2 & 0 & 0 & 0 & 0
\end{array}\right]^{\top}
\end{aligned}
$$

and the virtual frames are initialized standstill at

$$
\begin{aligned}
& \hat{\mathbf{q}}_{b, v}\left(t_{0}\right)=\hat{\mathbf{q}}_{I}+\varepsilon \frac{1}{2} \hat{\mathbf{q}}_{I} \otimes\left[\begin{array}{llll}
0 & 0 & 0 & -1
\end{array}\right]^{\top} \\
& \hat{\mathbf{q}}_{v, c}\left(t_{0}\right)=\hat{\mathbf{q}}_{I}+\varepsilon \frac{1}{2} \hat{\mathbf{q}}_{I} \otimes\left[\begin{array}{llll}
0 & 0 & 0 & 0
\end{array}\right]^{\top} .
\end{aligned}
$$

We employ a straight-line trajectory with a constant angular velocity reference, similar to that found in [7], defined as

$$
\begin{aligned}
\mathbf{p}_{d}^{n}(t) & =\left[\begin{array}{lll}
0 & ((75 / 4)-(3 / 4) t) 1 & -10
\end{array}\right]^{\top} \\
\boldsymbol{\omega}_{n d}^{d}(t) & =\left[\begin{array}{llll}
0 & 0 & 0 & 0.2
\end{array}\right]^{\top}
\end{aligned}
$$

with initial condition $\mathbf{q}_{n, d}=\mathbf{q}_{I}$. Figure 1 shows a side by side comparison of the dual quaternion error of system (34) on the left and (21) on the right, while Figure 2 shows a side by side comparison of the dual velocity error of system (34) on the left and (21) on the right. The simulations demonstrate the results of propositions 1 and 2 where it can be seen that the augmented system converges to the desired trajectory within roughly 25 seconds while the original system converges to a ball of radius $\gamma$ around the origin. Note that the attitude error and angular velocity error for system (21) does not converge to zero which is consistent with Remark (3). Moreover, Figure 3, shows top left the total error of system (34) and top right the total error of system (21). The total error of the augmented system goes towards zero while the total error of the real system enters a ball of radius $\gamma$. The plots middle left and right in Figure 3 shows the position error, measured in the inertial frame
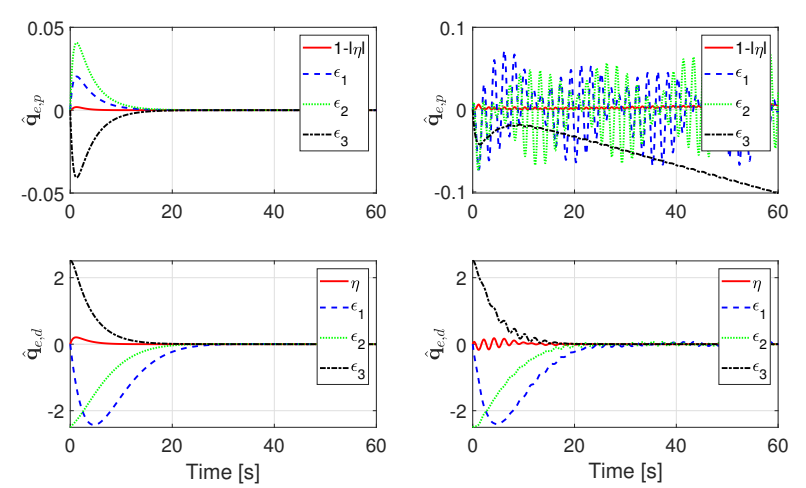

Fig. 1. Comparison dual quaternion error of system (34) ans (21)
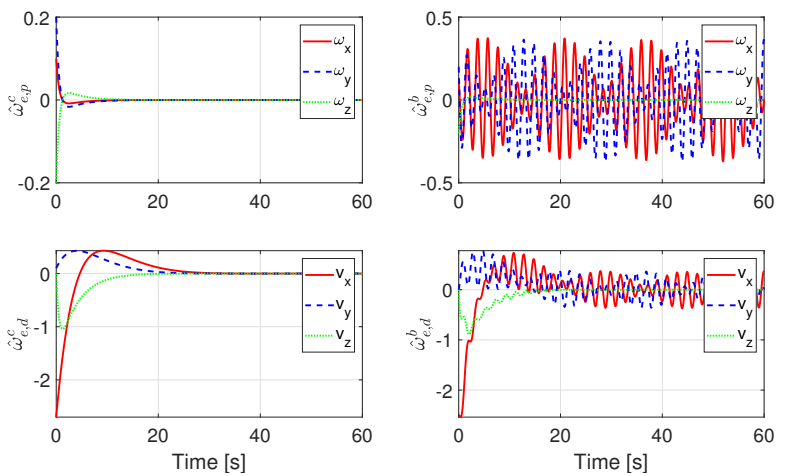

Fig. 2. Comparison dual velocity error of system (34) and (21)

$\mathcal{F}^{n}$, of systems (34) and (21) respectivly. The plot bottom left shows the attitude error of the body frame yaw angle and the desired yaw angle, while the bottom right plot show the applied torques. As the control strategy involves mapping the translational error onto the rotational actuators, this will, without saturation, cause large control effort and diminish the applicability of the method. It can be seen in the bottom right plot of Figure 3 the the inital torques are somewhat large, the size of which is directly affected by the size of $\Delta$ and the initial conditions. Moreover, due to the lack of damping, the torques does not become smaller as the system converges and the lack of damping becomes clear. As discussed in [15], non-linear systems with non-asymptotically stable zerodynamics are said to be strictly non-minimum phase. There are several ways to dampen the system, as will be examined in future work.
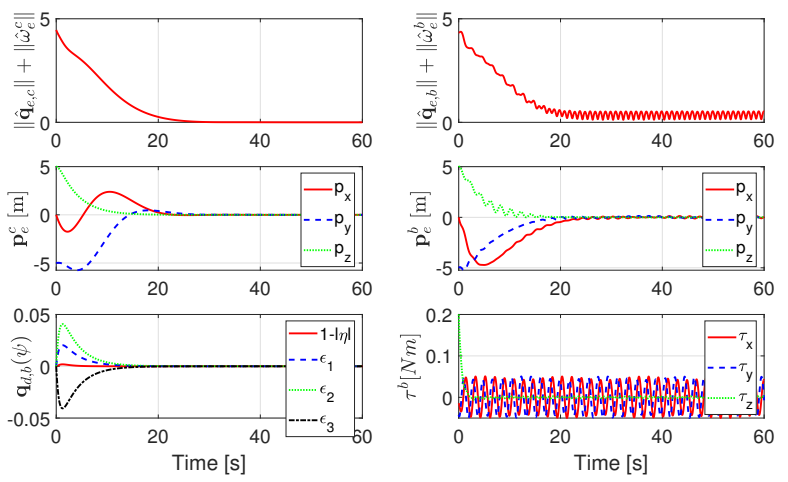

Fig. 3. Norm of dual error system, position error, yaw error and torques 


\section{CONCLUSION AND FUtURE WORK}

We proposed a new method to solve the trajectory tracking problem for the underactuated quadrotor platform, including a PD+ based state feedback control law for solving the tracking problem under this method. It was shown that the equilibria of the closed-loop augmented system are uniformly asymptotically stable, which implied that the equilibria of the closed-loop real system are practically asymptotically stable. Simulations demonstrate the theoretical results, however, they revealed that further work is necessary in order for the method to be implemented on a real quadrotor.

\section{APPENDIX}

\section{A. Proof of Lemma 3.1}

Proof: By direct calculation, and using the fact that $\mathbf{a}^{\top} \mathbf{S}(\mathbf{a})=0 \forall \mathbf{a} \in \mathbb{R}^{3}$, we have

$$
\begin{aligned}
& \hat{\mathbf{q}}_{e \pm}^{\top} \hat{\mathbf{T}}\left(\hat{\mathbf{q}}_{e \pm}\right)= \\
& \frac{1}{2}\left[\left(1 \mp \eta_{e}\right) \varepsilon_{e}^{\top} \eta_{e p} \varepsilon_{e p}^{\top}\right]\left[\begin{array}{cc} 
\pm \boldsymbol{\varepsilon}_{e}^{\top} & \mathbf{0}_{1 \times 3} \\
\eta_{e} \mathbf{I}+\mathbf{S}\left(\varepsilon_{e}\right) & \mathbf{0}_{3 \times 3} \\
-\boldsymbol{\varepsilon}_{e p}^{\top} & -\varepsilon_{e}^{\top} \\
\eta_{e p} \mathbf{I}+\mathbf{S}\left(\varepsilon_{e p}\right) & \eta_{e} \mathbf{I}+\mathbf{S}\left(\boldsymbol{\varepsilon}_{e}\right)
\end{array}\right] \\
& =\frac{1}{2}\left[\begin{array}{c} 
\pm\left(1 \mp \eta_{e}\right) \varepsilon_{e}^{\top}+\varepsilon_{e}^{\top}\left(\eta_{e} \mathbf{I}\right)-\eta_{e p} \boldsymbol{\varepsilon}_{e p}^{\top}+\varepsilon_{e p}^{\top}\left(\eta_{e p} \mathbf{I}\right) \\
-\eta_{e p} \boldsymbol{\varepsilon}_{e}^{\top}+\boldsymbol{\varepsilon}_{e p}^{\top}\left(\eta_{e} \mathbf{I}+\mathbf{S}\left(\boldsymbol{\varepsilon}_{e}\right)\right)
\end{array}\right]^{\top}
\end{aligned}
$$

It is trivial to see that the first part of the vector above reduces to $\pm \varepsilon_{e}^{\top}$. Further inspection of the second part reveals that by exchanging the dual part of the dual quaternion using the body frame convention in (11) we get

$$
\begin{aligned}
-\eta_{e p} \varepsilon_{e}^{\top}+\varepsilon_{e p}^{\top}\left(\eta_{e} \mathbf{I}+\mathbf{S}\left(\varepsilon_{e}\right)\right)= & \frac{1}{2}\left(\varepsilon_{e}^{\top} \mathbf{p}_{e}^{b} \varepsilon_{e}^{\top}\right. \\
& \left.+\eta_{e}^{2}\left(\mathbf{p}_{e}^{b}\right)^{\top}-\left(\mathbf{p}_{e}^{b}\right)^{\top} \mathbf{S}^{2}\left(\varepsilon_{e}\right)\right)
\end{aligned}
$$

which by the unit constraint of the primary part of $\hat{\mathbf{q}}_{e \pm}$ gives

$$
\frac{1}{2}\left(\varepsilon_{e}^{\top} \mathbf{p}_{e}^{b} \varepsilon_{e}^{\top}+\eta_{e}^{2}\left(\mathbf{p}_{e}^{b}\right)^{\top}-\left(\mathbf{p}_{e}^{b}\right)^{\top} \mathbf{S}^{2}\left(\varepsilon_{e}\right)\right)=\frac{1}{2}\left(\mathbf{p}_{e}^{b}\right)^{\top}
$$

Hence,

$$
\hat{\mathbf{q}}_{e \pm}^{\top} \hat{\mathbf{T}}\left(\hat{\mathbf{q}}_{e \pm}\right)=\frac{1}{2} \tilde{\varepsilon}^{\top}
$$

which concludes the proof.

\section{B. Proof of Lemma 3.2}

Proof: This lemma follows from the fact that a unit quaternion satisfies the property, $-c f$. [20]

$$
\begin{array}{ll}
0 \leq(1-\eta)^{2} \leq(1-\eta)(1+\eta)=1-\eta^{2}=\varepsilon^{\top} \varepsilon & \eta>0 \\
0 \leq(1+\eta)^{2} \leq(1-\eta)(1+\eta)=1-\eta^{2}=\varepsilon^{\top} \varepsilon & \eta<0
\end{array}
$$

By Lemma 3.1 we have that

$$
\Xi^{\top} \Xi=\frac{1}{4}\left(\varepsilon_{e}^{\top} \varepsilon_{e}+\frac{1}{4}\left(\left(\mathbf{p}_{e}^{b}\right)^{\top} \mathbf{p}_{e}^{b}\right)\right)
$$

while it may be shown that

$$
\hat{\mathbf{q}}_{e \pm}^{\top} \hat{\mathbf{q}}_{e \pm}=\left(\left(1 \mp \eta_{e}\right)^{2}+\varepsilon_{e}^{\top} \boldsymbol{\varepsilon}_{e}+\frac{1}{4}\left(\mathbf{p}_{e}^{b}\right)^{\top} \mathbf{p}_{e}^{b}\right)
$$

Then, by using (57), the proof is completed.

\section{REFERENCES}

[1] H. Gui and G. Vukovich, "Finite-time output-feedback position and attitude tracking of a rigid body," Automatica, vol. 74, pp. 270-278, 2016.

[2] N. A. Aspragathos and J. K. Dimitros, "A comparative study of three methods for robot kinematics," IEEE Transactions on Systems, Man, and Cybernetics, Part B (Cybernetics), vol. 28, no. 2, pp. 135-145, 1998.

[3] J. Funda and R. P. Paul, "A computational analysis of screw transformations in robotics," IEEE Transactions on Robotics and Automation, vol. 6, no. 3, pp. 348-356, 1990.

[4] H. T. Kussaba, L. F. Figueredo, J. Y. Ishihara, and B. V. Adorno, "Hybrid kinematic control for rigid body pose stabilization using dual quaternions," Journal of the Franklin Institute, vol. 354, no. 7, pp. 2769-2787, 2017.

[5] Y. D. Song and W. C. Cai, "New intermediate quaternion based control of spacecraft: part I-almost global attitude tracking," International Journal of Innovative Computing, Information and Control, vol. 8, no. 10, pp. 7307-7319, 2012.

[6] M.-D. Hua, T. Hamel, P. Morin, and C. Samson, "Introduction to feedback control of underactuated vtol-vehicles: A review of basic control design ideas and principles," IEEE Control Systems, vol. 33, no. 1, pp. 61-75, 2013.

[7] T. Lee, M. Leok, and N. H. McClamroch, "Nonlinear robust tracking control of a quadrotor UAV on SE(3)," Asian Journal of Control, vol. 15, no. 2, pp. 391-408, 2013.

[8] R. R. Warier, A. K. Sanyal, S. Sukumar, and S. P. Viswanathan, "Feedback tracking control schemes for a class of underactuated vehicles in SE (3)," in Proceedings of the American Control Conference, 2017, pp. 899-904, IEEE, 2017.

[9] T. S. Andersen, T.-A. Johansen, and R. Kristiansen, "Dual-quaternion backstepping control for a fully-actuated rigid-body," in Proceedings of the American Control Conference, pp. 5653-5658, IEEE, 2018.

[10] L. S. Mello, B. Aldorno, and G. V. Raffo, "Whole-body modeling and control of an unmanned aerial manipulator," in Proceedings of the XII Brazilian Symposium of Intelligent Automation. Natal, 2015.

[11] H. Abaunza, J. Cari, P. Castillo, R. Lozano, et al., "Quadrotor dual quaternion control," in Proceedings of the Workshop on Research, Education and Development of Unmanned Aerial Systems, pp. 195203, IEEE, 2015.

[12] H. Abaunza, P. Castillo, R. Lozano, and A. Victorino, "Quadrotor aerial manipulator based on dual quaternions," in Proceedings of the 2016 International Conference on Unmanned Aircraft Systems, pp. 152-161, IEEE, 2016.

[13] B. Paden and R. Panja, "Globally asymptotically stable 'PD+' controller for robot manipulators," International Journal of Control, vol. 47, no. 6, pp. 1697-1712, 1988.

[14] E. Oland, T. S. Andersen, and R. Kristiansen, "Underactuated control of quadrotors with collision avoidance," IFAC Proceedings Volumes, vol. 46, no. 30, pp. 162-167, 2013.

[15] J. M. Pflimlin, P. Souères, and T. Hamel, "Position control of a ducted fan VTOL UAV in crosswind," International Journal of Control, vol. 80, no. 5, pp. 666-683, 2007.

[16] H. Gui and G. Vukovich, "Dual-quaternion-based adaptive motion tracking of spacecraft with reduced control effort," Nonlinear Dynamics, vol. 83, no. 1-2, pp. 597-614, 2016.

[17] J. Sola, "Quaternion kinematics for the error-state KF," Laboratoire d'Analyse et d'Architecture des Systemes-Centre National de la Recherche Scientifique (LAAS-CNRS), Toulouse, France, Tech. Rep, 2012.

[18] R. Schlanbusch, A. Loria, R. Kristiansen, and P. J. Nicklasson, "PD+ based output feedback attitude control of rigid bodies," IEEE Transactions on Automatic Control, vol. 57, no. 8, pp. 2146-2152, 2012.

[19] H. K. Khalil, Nonlinear Systems. Prentice-Hall, 2002.

[20] R. Kristiansen, Dynamic synchronization of spacecraft. PhD thesis, Norwegian University of Science and Technology, 2008. 